\title{
ON LARGE DEVIATION PROBABILITIES IN THE CASE OF ATTRACTION TO A NON-NORMAL STABLE LAW
}

\author{
By C. C. HEYDE \\ University of Sheffield
}

$S U M M A R Y$. In this paper, the precise asymptotic behaviour of the large deviation probability is found in the case where the random variables are attracted to a non-normal stable law. This extends previous work of the same author in which only the order of magnitude of the large deviation probability was found.

\section{INTRODUCTION}

Let $X_{i}, i=1,2,3, \ldots$ be a sequence of independent and identically distributed random variables with law $\mathcal{L}(X)$ and write $S_{n}=\sum_{i=1}^{n} X_{i}$. Let $y_{n}, n=1,2,3, \ldots$ be a monotone sequence of positive numbers with $y_{n} \rightarrow \infty$ as $n \rightarrow \infty$ such that $y_{n}^{-1} S_{n} \stackrel{P}{\rightarrow} 0$. ( $P$ stands for convergence in probability). The probabilities $\operatorname{Pr}\left(\left|S_{n}\right|>y_{n}\right)$, or either of the one sided components, are called large deviation probabilities.

In the papers Heyde, (1967a and 1967b), the asymptotic behaviour of $\operatorname{Pr}\left(\left|S_{n}\right|>y_{n}\right)$ has been investigated for various types $X$ which are not attracted to the normal law. The results contained therein, however, have a shortcoming in that only the order of magnitude of the larger deviation probability is found and not the precise behaviour. It is the object of this paper to remedy this deficiency in the case of the (1967a) paper where the $X_{i}$ belong to the domain of attraction of a non-normal stable law. The context of the $(1967 \mathrm{~b})$ paper, namely with the $X_{i}$ not belonging to the domain of partial attraction of the normal distribution, is manifestly too general to allow for a corresponding complete answer.

\section{Details}

The formulation of the problem follows the same lines as that of Heyde (1967a) to which we refer for background details. Thus, the $X_{i}$ belong, with normalizing constants $B_{n}$, to the domain of attraction of the stable law with characteristic function

$$
\exp \left\{-a|t|^{\alpha}\left(1+i \frac{t}{|t|} \frac{c_{1}-c_{2}}{c_{1}+c_{2}} \tan \frac{\pi \alpha}{2}\right)\right\}
$$

$0<\alpha<2, \alpha \neq 1, a>0, c_{1}>0, c_{2}>0$. Certain rather exceptional cases ( $c_{1}$ or $c_{2}$ zero, $\alpha=1$ ) are excluded as a matter of convenience. If $\alpha>1$, we suppose that the $X_{i}$ are located so that $E X_{i}=0$. Consequently, we have $B_{n}^{-1} S_{n}$ converging in law to a stable distribution with characteristic function (1) and $x_{n}^{-1} B_{n}^{-1} S_{n} \stackrel{P}{\rightarrow} 0$ if $x_{n}$, $n=1,2,3, \ldots$ is a monotone sequence of positive numbers with $x_{n} \rightarrow \infty$ as $n \rightarrow \infty$. 


\section{SANKHYĀ : THE INDIAN JOURNAL OF STATISTICS : SERIES A}

Under the above conditions we may write for $x>0$, using Gnedenko and Kolmogorov (1954, Theorem 2, 175)

$$
\operatorname{Pr}(|X|>x)=\frac{M(x)}{x^{\alpha}}
$$

where $M(x)$ is a slowly varying function which satisfies the relation

$$
\frac{n M\left(B_{n}\right)}{B_{n}^{\alpha}} \rightarrow\left(c_{1}+c_{2}\right) \quad \text { as } n \rightarrow \infty .
$$

We shall establish the following theorem concerning the two sided large deviation probability. Corresponding one sided versions can probably be obtained in much the same manner but there are some technical difficulties to overcome.

Theorem : Let $\left\{x_{n}\right\}$ be a monotone sequence of positive numbers with $x_{n} \rightarrow \infty$ as $n \rightarrow \infty$. Then,

$$
\lim _{n \rightarrow \infty} \frac{\operatorname{Pr}\left(\left|S_{n}\right|>x_{n} B_{n}\right)}{n \operatorname{Pr}\left(|X|>x_{n} B_{n}\right)}=1
$$

or equivalently,

$$
\lim _{n \rightarrow \infty} \frac{\operatorname{Pr}\left(\left|S_{n}\right|>x_{n} B_{n}\right)}{\operatorname{Pr}\left(\max _{1 \leqslant k \leqslant n}\left|X_{k}\right|>x_{n} B_{n}\right)}=1 .
$$

This result extends that of Heyde (1967a) where it is shown, in essence, that

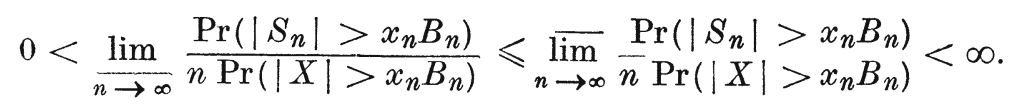

Proof: Firstly we note that the analysis of the theorem of Heyde (1967b) can be used and we deduce immediately that

$$
\lim _{n \rightarrow \infty} \frac{\operatorname{Pr}\left(\left|S_{n}\right|>x_{n} B_{n}\right)}{n \operatorname{Pr}\left(|X|>x_{n} B_{n}\right)} \geqslant 1
$$

It therefore just remains to show that

$$
\varlimsup_{n \rightarrow \infty} \frac{\operatorname{Pr}\left(\left|S_{n}\right|>x_{n} B_{n}\right)}{n \operatorname{Pr}\left(|X|>x_{n} B_{n}\right)} \leqslant 1
$$

and the result (4) will follow. The equivalence of the forms (4) and (5) is deduced precisely as in Heyde (1967b) and will not be repeated here.

For $1 \leqslant k \leqslant n$ and $n=1,2,3, \ldots$, define random variables

$$
X_{k n}=\left\{\begin{array}{cc}
X_{k} & \text { if } \quad\left|X_{k}\right| \leqslant z_{n} B_{n} \\
0 & \text { if } \quad\left|X_{k}\right|>z_{n} B_{n}
\end{array}\right.
$$




\section{ON LARGE DEVIATION PROBABILITIES}

where $z_{n}=x_{n}^{\gamma}, \quad 1>\gamma>1 / 2$, and write $S_{n n}=\sum_{k=1}^{n} X_{k n}$. Take $0<\varepsilon<1$ and define the events

$$
\begin{array}{ll}
E_{n}=\left\{\left|X_{k}\right|>(1-\varepsilon) x_{n} B_{n} \text { for at least one } k \leqslant n\right\}, \\
F_{n}=\left\{\left|X_{k}\right|>z_{n} B_{n} \quad \text { for at least two } k \text { 's } \leqslant n\right\}, \\
G_{n}=\left\{\left|S_{n n}\right|>\varepsilon x_{n} B_{n}\right\} .
\end{array}
$$

Then, we have

$$
\left\{\left|S_{n}\right|>x_{n} B_{n}\right\} \subset E_{n} U F_{n} U G_{n}
$$

so that

$$
\begin{aligned}
& \operatorname{Pr}\left(\left|S_{n}\right|>x_{n} B_{n}\right) \leqslant \operatorname{Pr}\left(E_{n}\right)+\operatorname{Pr}\left(F_{n}\right)+\operatorname{Pr}\left(G_{n}\right) \\
& \leqslant n \operatorname{Pr}\left(|X|>(1-\varepsilon) x_{n} B_{n}\right)+n^{2}\left[\operatorname{Pr}\left(|X|>z_{n} B_{n}\right)\right]^{2} \\
&+\operatorname{Pr}\left(\left|S_{n n}\right|>\varepsilon x_{n} B_{n}\right) .
\end{aligned}
$$

We shall deal separately with each of the three terms on the right hand side of (6).

From (2), we have

$$
\frac{n \operatorname{Pr}\left(|X|>(1-\varepsilon) x_{n} B_{n}\right)}{n \operatorname{Pr}\left(|X|>x_{n} B_{n}\right)}=\frac{M\left[(1-\varepsilon) x_{n} B_{n}\right]}{M\left[x_{n} B_{n}\right]} \cdot \frac{x_{n}^{\alpha} B_{n}^{\alpha}}{x_{n}^{\alpha} B_{n}^{\alpha}(1-\varepsilon)^{\alpha}} \rightarrow \frac{1}{(1-\varepsilon)^{\alpha}} \text { as } n \rightarrow \infty .
$$

Also,

$$
\begin{aligned}
\frac{n^{2}\left[\operatorname{Pr}\left(|X|>z_{n} B_{n}\right)\right]^{2}}{n \operatorname{Pr}\left(|X|>x_{n} B_{n}\right)} & =\frac{n M\left(B_{n}\right)}{B_{n}^{\alpha}} \cdot \frac{x_{n}^{\alpha}}{z_{n}^{2 \alpha}} \frac{\left[M\left(z_{n} B_{n}\right)\right]^{2}}{M\left(B_{n}\right) M\left(x_{n} B_{n}\right)} \\
& \leqslant C \frac{x_{n}^{\alpha}}{z_{n}^{2 \alpha}\left(\frac{x_{n}}{z_{n}}\right)^{\eta_{1}} z_{n}^{\eta_{2}}} \\
& =C x_{n}^{\alpha+\eta_{1}-\gamma\left(2 \alpha+\eta_{1}-\eta_{2}\right)}
\end{aligned}
$$

for $n$ sufficiently large using estimates obtained in Heyde (1967a), where $C$ is a positive constant and $\eta_{1}, \eta_{2}$ are arbitrarily small and positive. Since $1>\gamma>1 / 2$, we can choose $\eta_{1}$ and $\eta_{2}$ such that

$$
\alpha+\eta_{1}-\gamma\left(2 \alpha+\eta_{1}-\eta_{2}\right)<0
$$

and then

$$
\frac{n\left[\operatorname{Pr}\left(|X|>z_{n} B_{n}\right)\right]^{2}}{\operatorname{Pr}\left(|X|>x_{n} B_{n}\right)} \rightarrow 0 \text { as } n \rightarrow \infty
$$

Finally, it remains to consider $\operatorname{Pr}\left(\left|S_{n n}\right|>\varepsilon x_{n} B_{n}\right)$. We have, using Chebyshev's inequality,

$$
\begin{aligned}
\operatorname{Pr}\left(\left|S_{n n}\right|>\varepsilon x_{n} B_{n}\right) & \leqslant \varepsilon^{-2} x_{n}^{-2} B_{n}^{-2} E\left(S_{n n}^{2}\right) \\
& =\varepsilon^{-2} x_{n}^{-2} B_{n}^{-2}\left[n E X_{k n}^{2}+\frac{1}{2} n(n-1)\left(E X_{k n}\right)^{2}\right] .
\end{aligned}
$$




\section{SANKHYA : THE INDIAN JOURNAL OF STATISTICS : SERIES A}

Then,

$$
\begin{aligned}
\frac{E X_{k n}^{2}}{x_{n}^{2} B_{n}^{2} \operatorname{Pr}\left(|X|>x_{n} B_{n}\right)} & =\frac{|x| \stackrel{\int}{\leqslant} z_{n} B_{n}^{2} d \operatorname{Pr}(X \leqslant x)}{x_{n}^{2} B_{n}^{2} \operatorname{Pr}\left(|X|>x_{n} B_{n}\right)} \\
& \leqslant \frac{2 \int_{0}^{z_{n} B_{n}} x \operatorname{Pr}(|X|>x) d x}{x_{n}^{2} B_{n}^{2} \operatorname{Pr}\left(|X|>x_{n} B_{n}\right)} \\
& =\frac{2 \int_{0}^{z_{n} B_{n}} x \operatorname{Pr}(|X|>x) d x}{z_{n}^{2} B_{n}^{2} \operatorname{Pr}\left(|X|>z_{n} B_{n}\right)} \cdot \frac{z_{n}^{2} \operatorname{Pr}\left(|X|>z_{n} B_{n}\right)}{x_{n}^{2} \operatorname{Pr}\left(|X|>x_{n} B_{n}\right)}
\end{aligned}
$$

where we have used integration by parts to obtain the second last line. Furthermore, using standard properties of slowly varying functions (Feller, 1966, 273), we have

$$
\frac{2 \int_{0}^{z_{n} B_{n}} x \operatorname{Pr}(|X|>x) d x}{z_{n}^{2} B_{n}^{2} \operatorname{Pr}\left(|X|>z_{n} B_{n}\right)} \rightarrow \frac{2}{2-\alpha} \text { as } n \rightarrow \infty
$$

while

$$
\frac{z_{n}^{2} \operatorname{Pr}\left(|X|>z_{n} B_{n}\right)}{x_{n}^{2} \operatorname{Pr}\left(|X|>x_{n} B_{n}\right)}=\frac{z_{n}^{2-\alpha}}{x_{n}^{2-\alpha}} \frac{M\left(z_{n} B_{n}\right)}{M\left(x_{n} B_{n}\right)} \rightarrow 0 \text { as } n \rightarrow \infty
$$

and therefore,

$$
\frac{E X_{k n}^{2}}{x_{n}^{2} B_{n}^{2} \operatorname{Pr}\left(|X|>x_{n} B_{n}\right)} \rightarrow 0 \text { as } n \rightarrow \infty
$$

Also, if $\alpha<1$,

$$
\begin{aligned}
\left|E X_{k n}\right| \leqslant E\left|X_{k n}\right| & =\int_{|x|} \leqslant z_{n} B_{n}|x| d \operatorname{Pr}(X \leqslant x) \\
& \leqslant \int_{0}^{z_{n} B_{n}} \operatorname{Pr}(|X|>x) d x,
\end{aligned}
$$

using integration by parts, so that

$$
\frac{n\left(E X_{k n}\right)^{2}}{x_{n}^{2} B_{n}^{2} \operatorname{Pr}\left(|X|>x_{n} B_{n}\right)} \leqslant\left[\frac{\int_{0}^{z_{n} B_{n}} \operatorname{Pr}(|X|>x) d x}{z_{n} B_{n} \operatorname{Pr}\left(|X|>z_{n} B_{n}\right)}\right]^{2} \frac{n z_{n}^{2}\left[\operatorname{Pr}\left(|X|>z_{n} B_{n}\right)\right]^{2}}{x_{n}^{2} \operatorname{Pr}\left(|X|>x_{n} B_{n}\right)} .
$$

In this case,

$$
\frac{\int_{0}^{z_{n} B_{n}} \operatorname{Pr}(|X|>x) d x}{z_{n} B_{n} \operatorname{Pr}\left(|X|>z_{n} B_{n}\right)} \rightarrow \frac{1}{1-\alpha}
$$

while

$$
\frac{n z_{n}^{2}\left[\operatorname{Pr}\left(|X|>z_{n} B_{n}\right)\right]^{2}}{x_{n}^{2} \operatorname{Pr}\left(|X|>x_{n} B_{n}\right)}<\frac{n\left[\operatorname{Pr}\left(|X|>z_{n} B_{n}\right)\right]^{2}}{\operatorname{Pr}\left(|X|>x_{n} B_{n}\right)} \rightarrow 0 \text { as } n \rightarrow \infty
$$




\section{ON LARGE DEVIATION PROBABILITIES}

according to (8) above, so that for $\alpha<1$.,

$$
\frac{n\left(E X_{k n}\right)^{2}}{x_{n}^{2} B_{n}^{2} \operatorname{Pr}\left(|X|>x_{n} B_{n}\right)} \rightarrow 0 \text { as } n \rightarrow \infty
$$

If, on the other hand, $1<\alpha<2$, we have since $E X=0$,

$$
\begin{aligned}
\left|E X_{k n}\right| & =\left|\int_{|x| \leqslant z_{n} B_{n}} x d \operatorname{Pr}(X \leqslant x)\right| \\
& =\left|\int_{|x|>z_{n} B_{n}} x d \operatorname{Pr}(X \leqslant x)\right| \\
& \leqslant \int_{|x|>z_{n} B_{n}}|x| d \operatorname{Pr}(X \leqslant x) \\
& =-\int_{z_{n} B_{n}}^{\infty} x d \operatorname{Pr}(|X|>x) \\
& =\int_{z_{n} B_{n}}^{\infty} \operatorname{Pr}(|X|>x) d x+z_{n} B_{n} \operatorname{Pr}\left(|X|>z_{n} B_{n}\right),
\end{aligned}
$$

and

$$
\frac{\int_{z_{n} B_{n}}^{\infty} \operatorname{Pr}(|X|>x) d x}{z_{n} B_{n} \operatorname{Pr}\left(|X|>z_{n} B_{n}\right)} \rightarrow \frac{1}{\alpha-1},
$$

so that

$$
\begin{array}{r}
\frac{n\left(E X_{k n}\right)^{2}}{x_{n}^{2} B_{n}^{2} \operatorname{Pr}\left(|X|>x_{n} B_{n}\right)} \leqslant\left[\frac{\int_{n}^{\infty} B_{n} \operatorname{Pr}(|X|>x) d x}{z_{n} B_{n} \operatorname{Pr}\left(|X|>z_{n} B_{n}\right)}+1\right]^{2} \cdot \frac{n z_{n}^{2}\left[\operatorname{Pr}\left(|X|>z_{n} B_{n}\right)\right]^{2}}{x_{n}^{2} \operatorname{Pr}\left(|X|>x_{n} B_{n}\right)} \\
\rightarrow 0 \text { as } n \rightarrow \infty,
\end{array}
$$

again using (8). Then, from (9), (10), (11), and (12), we see that

$$
\frac{\operatorname{Pr}\left(\left|S_{n n}\right|>\varepsilon x_{n} B_{n}\right)}{n \operatorname{Pr}\left(|X|>x_{n} B_{n}\right)} \rightarrow 0 \text { as } n \rightarrow \infty \text {. }
$$

Finally, using (7), (8) and (13) in (6), we see that

$$
\varlimsup_{n \rightarrow \infty} \frac{\operatorname{Pr}\left(\left|S_{n}\right|>x_{n} B_{n}\right)}{n \operatorname{Pr}\left(|X|>x_{n} B_{n}\right)} \leqslant \frac{1}{(1-\varepsilon)^{2}}
$$

and since $\varepsilon, 0<\varepsilon<1$ is arbitrary,

$$
\varlimsup_{n \rightarrow \infty} \frac{\operatorname{Pr}\left(\left|S_{n}\right|>x_{n} B_{n}\right)}{n \operatorname{Pr}\left(|X|>x_{n} B_{n}\right)} \leqslant 1
$$

This furnishes the proof of the theorem. 


\section{SANKHYA : THE INDIAN JOURNAL OF STATISTICS : SERIES A}

\section{REFERENCES}

Feller, W. (1966) : An Introduction to Probability Theory and its Applications, Vol. II, Wiley, New York. Gmedenko, B. V. and Kolmogorov, A. N. (1954): Limit Distributions for Sums of Independent Random Variables, Translated and annotated by K. L. Chung, Addison Wesley, Cambridge (Mass.).

HEXDE, C. C. (1967a) : A contribution to the theory of large deviations for sums of independent random variables. Z. Wahrscheinlichkeitstheorie, 7, 303-308.

(1967b) : On large deviation problems for sums of random variables which are not attracted to the normal law. Ann. Math. Stat., 38, 1575-1578.

Paper received: June, $196 \%$. 